\title{
PREPARO DE MEDICAMENTOS ADMINISTRADOS VIA INTRAMUSCULAR NA PEDIATRIA: ATUAÇÃO DA EQUIPE DE ENFERMAGEM
}

\author{
Rafaela de Oliveira Mota ${ }^{1}$, Eva Anny Wélly de Souza Brito ${ }^{1}$, Thais Lima Vieira de Souza ${ }^{1}$, Laryssa Miranda \\ Vidal Cavalcante Farias², Érica Oliveira Matias ${ }^{3}$, Francisca Elisângela Teixeira Lima ${ }^{4}$
}

\begin{abstract}
RESUMO: Objetivou-se avaliar a atuação da equipe de Enfermagem na organização do ambiente, no preparo e na diluição de medicamentos administrados por via intramuscular na pediatria. Estudo observacional, quantitativo, desenvolvido mediante 327 observações do preparo de medicamento intramuscular, no período de dezembro de 2014 a maio de 2015, em um hospital pediátrico, Fortaleza-Ceará. Para observações utilizou-se um checklist composto pelas etapas: organização do ambiente com quatro ações; preparo e diluição de medicamentos com 12 ações. Na organização do ambiente, alcançou-se desempenho satisfatório na ação organizar a bancada, com $231(70,6 \%)$. A etapa de preparo e diluição do medicamento obteve desempenho satisfatório em seis ações: separar bolas de algodão com álcool e seca, com 252 (77\%); escolher seringa compatível com o volume a ser administrado, com $264(80,7 \%)$; selecionar adequadamente a agulha para aspiração, com $233(71,2 \%)$, e a agulha para administração, com 320 (97,8\%); trocar agulha depois do preparo, com 266 (81,3\%); e reconstituir medicação em água destilada, com 327 (100\%). Com isso, identificaram-se fragilidades como observar a data de validade do medicamento e identificar a medicação preparada que interferem na segurança da administração de medicamentos na pediatria.
\end{abstract}

DESCRITORES: Enfermagem; Injeções Intramusculares; Sistemas de Medicação no Hospital; Pediatria; Segurança do Paciente.

\section{PREPARATION OF INTRAMUSCULAR DRUGS IN PEDIATRICS: NURSING TEAM}

ABSTRACT: The objective of this study was to analyze work by a nursing team regarding the environmental organization, preparation and dilution of drugs administered intramuscularly in a pediatric setting. This was an observational and quantitative study developed through 327 observations of the preparation of intramuscular medication from December 2014 to May 2015 in a pediatric hospital in Fortaleza, in the state of Ceará. Observations employed a two-stage checklist: organization of the environment with four actions; preparation and dilution of medications with 12 actions. For organization of the environment, performance regarding the action of organizing the bench with a count of $231(70.6 \%)$ was satisfactory. The stage of medication preparation and dilution achieved satisfactory performance in six actions: separation of cotton balls soaked with alcohol from dry ones with 252 ( $77 \%$ ); selection of syringe compatible with the volume to be administered with $264(80.7 \%)$; proper selection of the needle for aspiration with 233 $(71.2 \%)$; needle for administration with $320(97.8 \%)$; change of needle after preparation with $266(81.3 \%)$; reconstitution of medication in distilled water with $327(100 \%)$. This allowed for the identification of weaknesses, such as observing the sell-by date of medications and identifying the medications prepared, actions that interfere with the safe administration of medications in pediatrics.

DESCRIPTORS: Nursing; Intramuscular Injections; Hospital Medication Systems; Pediatrics; Patient Safety.

\section{PREPARACIÓN DE MEDICAMENTOS DE ADMINISTRACIÓN INTRAMUSCULAR EN PEDIATRÍA: ACTUACIÓN DEL EQUIPO DE ENFERMERÍA}

RESUMEN: Se objetivó evaluar la actuación del equipo de Enfermería en la organización del ámbito, la preparación y dilución de medicamentos de administración intramuscular en pediatría. Estudio observacional, desarrollado sobre 327 observaciones de preparación de medicamentos intramusculares, entre diciembre de 2014 y mayo de 2015 en un hospital pediátrico de Fortaleza-Ceará. Se utilizó en las observaciones un check-list integrado por las etapas: organización del ámbito, con cuatro acciones; preparación y dilución de medicamentos, con 12 acciones. En la organización del ámbito se alcanzó desempeño satisfactorio en acción de organización de mesada, con 231 (70,6\%). La etapa de preparación y dilución del medicamento obtuvo desempeño satisfactorio en seis acciones: separar trozos de algodón con alcohol y secos, con $252(77 \%)$; elegir jeringa compatible con volumen a administrar, con $264(80,7 \%)$; selección adecuada de aguja para aspiración, con 320 (97,8\%); cambiar aguja luego de preparación, con 266 $(81,3 \%)$; y reconstitución de medicación con agua destilada, con $327(100 \%)$. Así, fueron identificadas debilidades, como observar fecha de vencimiento del medicamento e identificar la medida preparada, que interfieren con la seguridad de administración de medicamentos en pediatría.

DESCRIPTORES: Enfermería; Inyecciones Intramusculares; Sistemas de Medicación en Hospital; Pediatría; Seguridad del Paciente.

${ }^{1}$ Discente de Enfermagem. Universidade Federal do Ceará. Fortaleza, CE, Brasil.

${ }^{2}$ Enfermeira. Residente em Enfermagem Obstétrica. Universidade Federal do Ceará. Fortaleza, CE, Brasil.

${ }^{3}$ Enfermeira. Doutoranda em Enfermagem. Universidade Federal do Ceará. Fortaleza, CE, Brasil..

${ }^{4}$ Enfermeira. Doutora em Enfermagem. Docente de Enfermagem da Universidade Federal do Ceará. Fortaleza, CE, Brasil.

Autor Correspondente:

Francisca Elisângela Teixeira Lima

Universidade Federal do Ceará

R. Alexandre Baraúna, 1115 - 60430-160 - Fortaleza, CE, Brasil

E-mail: felisangela@yahoo.com.br
Recebido: 01/03/2016 Finalizado: 01/08/2016 


\section{- INTRODUÇÃO}

A segurança do paciente envolve a ação de evitar erros durante a prestação de cuidados, com a finalidade de eliminar agravos acarretados por tais falhas. Para isso, é necessário que os profissionais de saúde desenvolvam comportamentos, como: liderança, trabalho em equipe, comunicação, aprender com os erros, justiça, cuidado centrado no paciente e prática baseada em evidências ${ }^{(1)}$.

No Brasil, o enfermeiro é o profissional responsável pelo processo de administração de medicamentos, constituindo-se no líder da equipe de Enfermagem e assumindo papel fundamental tanto no cuidado ao paciente que se encontra em terapia medicamentosa quanto na disseminação do conhecimento acerca desta prática para a equipe ${ }^{(2)}$.

A Lei $n^{\circ} 7.498$ regulamenta a Enfermagem e suas atividades auxiliares, afirmando que estas somente podem ser exercidas por pessoas legalmente habilitadas e inscritas no Conselho Regional de Enfermagem com jurisdição na área onde ocorre o exercício. Ainda de acordo com esta lei, planejamento, direção e execução do programa de Enfermagem fazem parte do exercício da profissão, além dos cuidados diretos de Enfermagem aos pacientes graves com risco de morte, que exigem habilidade técnica, embasamento científico e capacidade de tomada de decisões imediatas ${ }^{(3)}$.

Dentre as vias de administração de medicamentos utilizadas, a intramuscular (IM) é uma das mais realizadas, devido a sua rápida velocidade de absorção da solução administrada, perdendo apenas para a via endovenosa, que tem ação imediata. A utilização da via intramuscular torna-se mais evidente pelo fato da via endovenosa ser mais difícil, devido ao desafio de manter estes cateteres por períodos mais prolongados, diminuindo possíveis complicações relacionadas à terapia medicamentosa ${ }^{(4)}$.

Por se tratar de um processo de alta complexidade, quando não planejado, controlado e monitorado por meio de indicadores, fica exposto à imprevisibilidade de seus resultados, interferindo na qualidade da assistência. Além disso, o elevado número de medicamentos administrados aos pacientes aumenta a vulnerabilidade dos pacientes aos riscos ${ }^{(5)}$.

Os riscos mais evidenciados que envolvem o processo de administração de medicamentos por meio da via IM são: dor persistente no local, endurecimento, diminuição da sensibilidade, dano tecidual (lesão de nervos), hematomas, abscessos, reações alérgicas, lesões de necrose tecidual, contratura de grupos musculares, fibrose e até perda de amplitude de movimentos articulares ${ }^{(6-7)}$.

Neste contexto, o processo de administração de medicamentos por meio da via IM na pediatria exige dos profissionais envolvidos conhecimentos sobre o preparo correto, visto que esses pacientes constituem um grupo vulnerável, possuindo características farmacocinéticas e farmacodinâmicas com modificações rápidas ao longo do desenvolvimento infantil(8-9).

Portanto, para melhor aplicabilidade do processo de preparo de medicamentos intramusculares na pediatria, é necessário responder aos seguintes questionamentos: como ocorre a organização do ambiente, o preparo e a diluição de medicamentos para ser administrados pela via IM em crianças?

Acredita-se que a prática de Enfermagem na execução do preparo de medicamentos pela via IM na pediatria apresenta divergências com a técnica recomendada pela literatura científica, no que se refere à seleção do material e diluição da medicação, como fazer a identificação da medicação e verificar a validade do medicamento ${ }^{(10)}$.

Este estudo é de suma importância para todos que trabalham com administração de medicamentos pela via IM e para os demais profissionais da área da saúde, pois visa identificar as possíveis falhas durante o preparo do medicamento que possam interferir na segurança do paciente, o que possibilita uma posterior correção e melhoria desse processo, a fim de eliminar tais falhas, evitando a ocorrência do erro novamente.

Assim, o presente estudo tem como objetivo avaliar a atuação da equipe de Enfermagem na organização do ambiente, no preparo e na diluição de medicamentos administrados por via intramuscular na pediatria. 
Trata-se de um estudo observacional, transversal, de abordagem quantitativa, desenvolvido em um hospital pediátrico da rede municipal de Fortaleza-Ceará. Tal hospital possui seis enfermarias para atendimento clínico de crianças e adolescentes (3 meses a 18 anos incompletos), com funcionamento 24 horas diárias.

Participaram do estudo 11 profissionais de Enfermagem que atuavam na unidade de administração de medicamentos por via intramuscular da referida instituição, sendo um enfermeiro e dez técnicos de enfermagem, que atenderam aos seguintes critérios de inclusão: ser profissional de enfermagem que atue no processo de administração de medicamento por via intramuscular e estar em serviço nos dias que foram realizadas as observações. Já os critérios de exclusão foram: estar de férias, licença ou afastado de suas atividades no período da coleta de dados.

A coleta de dados foi realizada no período de dezembro de 2014 a maio de 2015, nos turnos manhã (8 às 12 horas), tarde (13 às 17 horas) ou noite (18 às 22 horas), em dias aleatórios, contemplando seis turnos por semana. Para coleta, realizaram-se 327 observações sistemáticas do preparo do medicamento administrado por via intramuscular em crianças atendidas na referida instituição que atenderam aos seguintes critérios de inclusão: ter idade $<10$ anos; ter prescrição médica de medicamento para administração pela via intramuscular; e ser realizada a administração do medicamento pela via intramuscular na referida instituição. Já os critérios de exclusão foram: apresentar um quadro clínico de emergência, por exemplo, convulsão.

O instrumento do tipo checklist utilizado para a observação sistemática do preparo do medicamento foi um formulário contendo os dados de identificação das crianças (sexo, peso, idade e temperatura corporal), sendo estes coletados por meio da leitura da prescrição médica; e os dados referentes às duas etapas: 1) etapa de organização do ambiente para o preparo dos medicamentos; e 2) etapa do preparo e diluição do medicamento para administração pela via intramuscular.

A observação foi realizada por três acadêmicos de Enfermagem que receberam instruções prévias acerca do instrumento e das precauções no momento da coleta, como a não intervenção no momento do procedimento para evitar interferência na pesquisa.

Para observação, uma das acadêmicas ficava na unidade no dia e horário estabelecido aguardando a ocorrência do evento. Diante da chegada da criança, solicitou-se a assinatura do termo de consentimento livre e esclarecido, para observar o procedimento.

Utilizou-se um instrumento do tipo checklist, que continha as ações desenvolvidas no processo de administração de medicamento por via intramuscular, contemplando a organização do ambiente, preparo e diluição de medicamentos. O instrumento foi validado por especialistas na área de saúde da criança quanto à relevância e à clareza dos itens com Índice de Validade de Conteúdo (IVC) superior a 0,8 .

A etapa de organização do ambiente para o preparo dos medicamentos foi constituída por quatro ações: realizar limpeza da bancada; organizar a bancada; dispor de bandeja ou cuba rim; usar equipamentos de proteção individual.

A etapa do preparo e diluição do medicamento foi constituída por doze ações: conferir o rótulo da medicação com a prescrição; observar a data da validade do medicamento; verificar a integridade dos invólucros; inspecionar o frasco para observar possíveis partículas, alteração de cor, rachaduras ou vazamentos; fazer desinfecção da ampola ou frasco-ampola; separar bolas de algodão com álcool e seca; escolher seringa compatível com o volume a ser administrado; selecionar adequadamente agulhas para aspiração; selecionar adequadamente agulhas para administração IM; trocar agulha depois do preparo (se necessário); reconstituir medicação em água destilada (AD) respeitando os princípios de prevenção da infecção hospitalar (se necessário); e identificar a medicação preparada.

Os dados coletados na pesquisa foram armazenados em um banco de dados produzidos pelo Excel do Windows 2010, processados e analisados com a estatística descritiva e de acordo com a literatura pertinente à temática. Para a análise de desempenho dos profissionais em cada etapa do processo de 
administração de medicamento por via intramuscular, foi adotado como satisfatório o desempenho cujo ponto de corte foi igual ou superior a $70 \%^{(11-12)}$.

A realização do estudo seguiu os preceitos éticos, respeitando as normas da Resolução $n^{\circ} 466 / 2012^{(13)}$, do Conselho Nacional de Saúde, sendo aprovado pelo comitê de ética sob parecer $\mathrm{n}^{\circ}$ 805.953. Todos os profissionais de enfermagem e os acompanhantes foram orientados quanto aos objetivos do estudo e concordaram em ser observados durante a realização do processo de administração de medicamento intramuscular, assinando o termo de consentimento livre e esclarecido.

\section{- RESULTADOS}

O perfil das crianças que foram observados o preparo dos medicamentos administrados por via intramuscular apresentou as seguintes características: 186 (56,9\%) eram do sexo masculino, com média de idade de 3,4 anos, prevalecendo crianças classificadas como toddler com 147 (44,9\%), compreendendo a faixa etária de 12 a 36 meses, e 229 (70,2\%) crianças apresentavam hipertermia.

A Tabela 1 apresenta as informações sobre a organização do ambiente para o preparo dos medicamentos administrados por via intramuscular.

Tabela 1 - Distribuição do número de observações segundo as ações da etapa organização do ambiente para o preparo dos medicamentos. Fortaleza, CE, Brasil, 2015

\begin{tabular}{lcc} 
Ações & N & \% \\
\hline Realizar limpeza da bancada & 93 & 28,4 \\
\hline Organizar a bancada & 231 & 70,6 \\
\hline Dispor de bandeja ou cuba rim & 185 & 56,5 \\
\hline Usar equipamento de proteção individual & 195 & 59,6
\end{tabular}

Para a etapa organização do ambiente para o preparo dos medicamentos composta por 4 ações, alcançou-se um desempenho satisfatório com percentual $>70 \%$ em uma ação, compreendendo $25 \%$ do total de ações dessa etapa.

Conforme a Tabela 1, em $93(28,4 \%)$ das observações, os profissionais realizaram a limpeza da bancada, enquanto que em $231(70,6 \%)$ realizaram a organização do material sob a mesma para posterior preparação do medicamento.

Em 185 (56,5\%) observações, identificou-se que os profissionais dispuseram de bandeja ou cuba rim para levar a medicação até o local onde a criança se encontrava, enquanto que nas demais vezes o profissional levava os medicamentos preparados em mãos.

Para a ação usar equipamento de proteção individual, identificou-se que em 195 (59,6\%) das observações foram utilizados gorro e máscara. De forma parcial, o gorro foi o equipamento de proteção individual utilizado na maioria das observações com 192 (58,7\%). No caso das luvas, em nenhuma das observações foi detectado o seu uso.

A Tabela 2 apresenta as informações sobre as ações da etapa do preparo e diluição dos medicamentos administrados por via intramuscular.

Para a etapa de preparo dos medicamentos composta por 12 ações, alcançou-se um desempenho satisfatório com percentual > 70\% em 6 ações, compreendendo 50\% do total de ações dessa etapa.

As ações conferir o rótulo da medicação, observar a data de validade, verificar a integridade dos invólucros, inspecionar o frasco e fazer sua desinfecção obtiveram 128 (39,1\%), 17(5,1\%), 34(10,3\%), 86 $(26,2 \%)$ e $19(5,8 \%)$, respectivamente.

Quanto à separação de bolas de algodão com álcool e secas, notou-se que em $77 \%(n=252)$ das observações foi realizada, sendo que em apenas $16,8 \%(n=55)$ dos procedimentos esta foi feita de 
Tabela 2 - Distribuição do número de observações segundo as ações da etapa do preparo e diluição dos medicamentos. Fortaleza, CE, Brasil, 2015

\begin{tabular}{lcc} 
Ações & $\mathbf{N}$ & $\mathbf{\%}$ \\
\hline Conferir o rótulo da medicação com a prescrição & 128 & 39,1 \\
\hline Observar a data de validade do medicamento & 17 & 5,1 \\
\hline Verificar a integridade dos invólucros & 34 & 10,3 \\
\hline $\begin{array}{l}\text { Inspecionar o frasco para observar possíveis partículas, alteração da cor, rachaduras } \\
\text { ou vazamentos }\end{array}$ & 86 & 26,2 \\
\hline Fazer desinfecção da ampola ou frasco-ampola & 19 & 5,8 \\
\hline Separar bolas de algodão com álcool e seca & 252 & 77 \\
\hline Escolher seringa compatível com o volume a ser administrado & 264 & 80,7 \\
\hline Selecionar adequadamente a agulha para aspiração & 233 & 71,2 \\
\hline Selecionar adequadamente a agulha para administração IM & 320 & 97,8 \\
\hline Trocar agulha depois do preparo (se necessário) & 266 & 81,3 \\
\hline $\begin{array}{l}\text { Reconstituir medicação em AD, respeitando os princípios de prevenção de infecção } \\
\text { hospitalar (se necessário) }\end{array}$ & 327 & 100 \\
\hline Identificar a medicação preparada & 17 & 5,1
\end{tabular}

forma completa, em que a grande parcela, 60,2\% ( $n=197)$, desempenhava esta etapa de forma parcial, principalmente separando apenas bolas de algodão com álcool.

A ação escolher seringa compatível com o volume a ser administrado foi observado em 80,7\% $(n=264)$ dos preparos. Em relação à ação selecionar adequadamente agulhas para aspiração, a agulha ideal é a 40x12, porém a instituição só dispusera de agulha 25x8 e 25x7, sendo que esse fator não interfere no preparo do medicamento, apenas vai prolongar o tempo de preparo. A ação selecionar adequadamente agulhas para administração IM, foi visualizada em 97,8\% $(n=320)$ das observações. Quanto à troca da agulha, quando necessário, foi observada esta realização em $81,3 \%(\mathrm{n}=226)$ dos casos.

Ao observar se a reconstituição do medicamento em água destilada (AD), notou-se que em $100 \%$ das observações a mesma estava sendo preparada satisfatoriamente, respeitando os princípios de prevenção de infecção hospitalar.

Em relação à ação identificar a medicação preparada, em 5,1\% $(n=17)$ das observações foi constatada que tal ação foi executada, sendo de suma importância para garantir a segurança ao paciente, a fim de evitar possíveis erros durante a administração de medicamentos.

\section{DISCUSSÃO}

As discussões estão expostas conforme a sequência das ações realizadas para organização do ambiente, preparo e diluição de medicamentos pela via intramuscular na criança.

Com relação à etapa da organização do ambiente para o preparo dos medicamentos administrados por via intramuscular, ressalta-se a importância quanto ao seguimento das recomendações para as ações com a finalidade de garantia da segurança microbiológica e implicações para a segurança do paciente $\mathrm{e}^{(14)}$.

A segurança microbiológica fica comprometida quando a equipe de Enfermagem no processo de administração de medicamentos não adota as seguintes ações: limpeza da bancada antes e após o preparo da medicação, não uso de máscara durante o preparo das soluções, não realizar a desinfecção de frascos e ampolas, entre outros aspectos que potencializam os $\operatorname{riscos}^{(14)}$.

É relevante que durante o ato de preparar o material e as soluções de forma asséptica, a equipe de Enfermagem se atente para que essas tarefas sejam realizadas de forma correta. Desinfecção do material e da bancada, organização e seleção do material adequado para o procedimento, preparo do 
medicamento, inspeção das soluções quanto à presença de partículas, corpo estranho, mudança de coloração e validade, disposição organizada de todo o material, são imprescindíveis para o desempenho do procedimento de administrações de injeções intramusculares com êxito.

No presente estudo não se obteve um desempenho satisfatório na ação fazer a desinfecção da bancada, apresentando apenas $28,4 \%$ das observações e desinfecção dos frascos e ampolas em 5,8\%. Corroborando com uma pesquisa desenvolvida em um hospital público municipal da rede sentinela do Rio de Janeiro, com pacientes críticos alocados em clínicas e que são submetidos à terapia com múltiplos fármacos, ao constatar que em $77,26 \%$ dos preparos observados não houve desinfecção de bancada e em $80,27 \%$ não houve desinfecção das ampolas e frascos ${ }^{(14)}$.

Para a realização da desinfecção, recomenda-se o uso do álcool a $70 \%$ com a finalidade de reduzir a carga microbiológica, pois possui como vantagem ser um produto de baixo custo e fácil aplicação ${ }^{(15)}$. O álcool $70 \%$ é um dos principais desinfetantes utilizados em serviços de saúde e pode ser aplicado em superfícies ou artigos por meio de fricção ${ }^{(16)}$.

Outra ação importante para a segurança do paciente é o uso do equipamento de proteção individual durante o preparo do medicamento. Diante dos equipamentos de proteção, destaca-se a luva de procedimento pelas seguintes razões: reduzir o risco de contaminação das mãos dos profissionais de saúde com sangue e outros fluidos corporais; reduzir o risco de disseminação de germes para o ambiente; e proteger os profissionais e os pacientes do risco de infecção cruzada ${ }^{(17)}$.

Entretanto, há algumas condições em que não é preconizado o uso de luvas, tais como: aplicar injeções intradérmicas, subcutâneas e intramusculares de rotina; se a pele do profissional de saúde estiver intacta; se a pele do paciente estiver intacta ${ }^{(18)}$.

A conferência do rótulo da medicação com a prescrição foi constatada em 39,1 \% das observações. É fundamental ler os rótulos dos medicamentos; conferir com a prescrição médica; identificar o medicamento com o seu próprio nome conferir o nome do paciente, o número do leito, o nome do profissional responsável, a via de administração, a dose adequada e o horário certo são fatores que diminuem os erros por falhas de comunicação. Além disso, conferir a verbalização do paciente e com a sua pulseira de identificação, para certificar-se que está diante do paciente correto $^{(19)}$.

Erros encontrados nas prescrições podem ser classificados como erros de redação, sendo os mais prevalentes os problemas com a forma farmacêutica, a omissão da dose e a via de administração ${ }^{(20)}$. Destaca-se que omitir um erro impossibilita a implementação de intervenções, tanto que previnam ou tratem consequências para o paciente, quanto que impeçam sua ocorrência novamente ${ }^{(8)}$.

Em um estudo realizado em uma unidade hospitalar pediátrica em Minas Gerais, foi observado que em $5 \%$ dos casos não houve consulta à prescrição antes do preparo; que em $42 \%$ foi utilizada técnica incorreta de preparo; que em $53,2 \%$ a higienização das mãos apresentou falhas; que em $31,2 \%$ houve déficit na desinfecção de frasco-ampola; que em $12,5 \%$ houve contaminação de materiais e medicamentos; e que em 3,1\% houve reutilização de materiais ${ }^{(21)}$.

Em um estudo sobre a antissepsia para administração de medicamentos por via endovenosa e intramuscular, foi observado que em $40,5 \%$ das administrações por via endovenosas e em $37,5 \%$ das administrações intramusculares foram realizados cinco ou mais movimentos, em um mesmo sentido, com o algodão embebido com álcool a $70 \%$, sendo este o procedimento preconizado para a realização da antissepsia da pele e/ou desinfecção do injetor de borracha ${ }^{(22)}$.

Outro aspecto a ser considerado é o comprimento da agulha a ser utilizada, que deve ser suficiente para penetrar no tecido subcutâneo e depositar a medicação no corpo do músculo. Agulhas com pequeno diâmetro (calibre 25 a 30) provocam o menor desconforto, contudo, diâmetros maiores são necessários para medicações mais viscosas ${ }^{(23)}$.

Diante dos resultados encontrados, a ação selecionar adequadamente a agulha para administração IM requer um conhecimento do profissional para avaliar o paciente quanto à distribuição da massa muscular do paciente e características do medicamento como viscosidade evolume a ser administrado ${ }^{(24)}$.

A troca da agulha após o preparo da medicação e antes da administração do medicamento é de suma importância quando se visa à segurança do paciente, assim se mantém a integridade do sistema 
infusional, garantindo a não exposição de contaminação por meio de agentes externos ${ }^{(25)}$.

No processo de reconstituição ou diluição de medicamentos, é importante que se pondere aspectos como a hora do preparo e o prazo de estabilidade pós-reconstituição/ diluição, considerando que pode se alterar quando o profissional prepara o medicamento com muita antecedência ao horário em que será administrado, ou prepara os medicamentos sem observar protocolos de diluição. Esses fatores estão frequentemente presentes no cotidiano da equipe de enfermagem e acabam por dificultar a prevenção do erro e a garantia da segurança na terapia medicamentosa ${ }^{(14)}$.

Com relação à ação identificar a medicação preparada, foi observado que 5,1\% foi realizada de forma satisfatória. Destaca-se que um estudo desenvolvido sobre os erros na administração de medicamentos constatou que a identificação se faz necessária no processo de preparo e administração de medicamento, preconizando a existência de alguns dados, como o nome e o leito do paciente, com a finalidade de minimizar os erros no processo de administração de medicamentos ${ }^{(26)}$.

Destaca-se que a ação transportar medicamentos manualmente representa risco de acidentes, contaminação da solução e interferência na segurança. Ressalta-se a necessidade de cuidados para manter a integridade físico-química e microbiológica dos medicamentos, com o uso de bandejas ou cuba-rim ${ }^{(27)}$.

\section{CONCLUSÃO}

O presente estudo permitiu avaliar as ações realizadas pelos profissionais de enfermagem durante o preparo de medicamentos administrados por via intramuscular na pediatria, de acordo com os seguintes achados: desempenho satisfatório quanto à limpeza da bancada, escolha da seringa compatível com o volume a ser administrado, seleção adequada da agulha para aspiração, bem como para a administração. Já para o uso completo de equipamento de proteção individual recomendado para a realização do procedimento, observação da data de validade do medicamento e desinfecção da ampola ou frasco-ampola, foi observado desempenho insatisfatório.

Dessa forma, possibilitou-se realizar um diagnóstico situacional da equipe de enfermagem na atuação das etapas de organização do ambiente e preparo e diluição de medicamentos administrados por via intramuscular na pediatria, que permitirá o aperfeiçoamento da qualidade da assistência a saúde, contribuindo para a resolução dos problemas identificados, por meio da efetivação de práticas eficazes.

Portanto, destaca-se a necessidade da realização de novos estudos, em outros contextos, com metodologias de avaliação diferenciadas com a finalidade de tornar o processo de administração de medicamentos na pediatria mais seguro.

\section{REFERÊNCIAS}

1. Harada MJCS, Pedreira MLG. O erro humano e sua prevenção. In: Agência Nacional de Vigilância Sanitária (ANVISA). Assistência Segura: Uma Reflexão Teórica Aplicada à Prática. Brasília: ANVISA; 2013. p. 41-55.

2. Azevedo Filho FMA, Martins IMS, Soares CSRS, Fazendeiro PG, Paranaguá TTB, Bezerra ALQ. Administração de medicamentos: conhecimento de enfermeiros do setor de urgência e emergência. Enferm. glob. [Internet] 2012; 11(26) [acesso em 17 mai 2016]. Disponível: http://dx.doi.org/10.4321/S1695-61412012000200005

3. Brasil. Lei n. 7.498, de 25 de junho de 1986. Dispõe sobre a Regulamentação do Exercício da Enfermagem e dá outras providências. Diário Oficial da República Federativa do Brasil, Brasília, 25 jun. 1986. Seção 1:1.

4. Ferreira FLC, e Silva GF, da Fonseca PML, Christoffel MM. Terapia intravenosa em neonatologia e na pediatria: uma revisão sistemática da literatura. R.pesq.: cuid.fundam. online. [Internet] 2010; 2(Ed. Supl) [acesso em 17 mai 2016]. Disponível: http://dx.doi.org/10.9789/2175-5361.2010.v0i0.\%25p

5. Silva AEBC. Análise de risco do processo de administração de medicamentos por via intramuscular em pacientes de um Hospital Universitário de Goiás [tese]. Ribeirão Preto (SP): Escola de Enfermagem de Ribeirão Preto; 2008. 
6. Cançado RD, Lobo C, Friedrich JR. Tratamento da anemia com ferro ferropriva com ferro por via parenteral. Rev. Bras. Hematol. Hemoter. [Internet] 2010; 32(2) [acesso em 17 mai 2016]. Disponível:

http://dx.doi.org/10.1590/S1516-84842010005000066

7. Portela JL, Piva JP. Midazolam versus diazepam para tratamento de estado de mal epiléptico em emergência pediátrica. Scientia Medica. [Internet] 2011; 21(4) [acesso em 17 mai 2016]. Disponível:

http://revistaseletronicas.pucrs.br/ojs/index.php/scientiamedica/article/view/9176/7244

8. Belela ASC, Peterlini MAS, Pedreira MLG. Revelação da ocorrência de erro de medicação em unidade de cuidados intensivos pediátricos. Rev. bras. ter. intensiva. [Internet] 2010; 22(3) [acesso em 17 mai 2016]. Disponível: http://dx.doi.org/10.1590/S0103-507X2010000300007

9. Tonello P, Andriguetti LH, Perassolo MS, Ziulkoski AL. Avaliação do uso de medicamentos em uma unidade pediátrica de um hospital privado do sul do Brasil. RevCiêncFarm Básica Apl. [Internet] 2013; 34(1) [acesso em 17 mai 2016]. Disponível: http://serv-bib.fcfar.unesp.br/seer/index.php/Cien_Farm/article/view/2150/1369

10. Hospital São Paulo. Sistema de Gestão de Qualidade. Procedimento Operacional Padrão: Preparo e administração de medicamento por via intramuscular (IM). Associação Paulista para o Desenvolvimento da Medicina - São Paulo, 2015. [acesso em 17 mai 2016]. Disponível:

http://www.hospitalsaopaulo.org.br/sites/manuais/arquivos/2015/POP_Prep_e_adm_via_IM.pdf

11. Torres MM, Andrade D, Santos CB. Punção venosa periférica: avaliação de desempenho dos profissionais de enfermagem. Rev Latino -AmEnferm. [Internet] 2005; 13(3) [acesso em 17 mai 2016]. Disponível:

http://www.revistas.usp.br/rlae/article/view/2085/2170

12. Peduzzi M, Anselmi ML, França Júnior I, dos Santos CB. Qualidade no desempenho de técnicas dos trabalhadores de enfermagem de nível médio. RevSaúde Pública. [Internet] 2006; 40(5) [acesso em 28 fev 2016]. Disponível: http://dx.doi.org/10.1590/S0034-89102006000600014

13. Brasil. Resolução no 466/12 de 12 de dezembro de 2012. Diretrizes e normas regulamentadoras de pesquisas envolvendo seres humanos. Conselho Nacional de Saúde, Brasília, 12 dez. 2012.

14. Camerini FG, da Silva LD. Segurança do paciente: análise do preparo de medicação intravenosa em hospital da rede sentinela. Texto contexto-enferm. [Internet] 2011; 20(1) [acesso em 28 fev 2016]. Disponível:

http://dx.doi.org/10.1590/S0104-07072011000100005

15. Graziano MU, Graziano KU, Pinto FMG, Bruna CQM, de Souza RQ, Lascala CA. Eficácia da desinfecção com álcool 70\% (p/v) de superfícies contaminadas sem limpeza prévia. Rev. Latino-Am. Enfermagem. [Internet] 2013; 21(2) [acesso em 28 fev 2016]. Disponível: http://dx.doi.org/10.1590/S0104-11692013000200020

16. Assad C, Reinehr E, Siliprandi EMO, Costa G. Limpeza e desinfecção de superfícies. In: Agência Nacional de Vigilância Sanitária (ANVISA). Segurança do paciente em serviços de saúde: limpeza e desinfecção de superfícies. Brasília: ANVISA; 2010. p. 72.

17. Agência Nacional de Vigilância Sanitária (ANVISA). Luvas cirúrgicas e luvas de procedimentos: considerações sobre o seu uso. Boletim Informativo de Tecnovigilância- Brasília. 2011. [acesso em 28 fev 2016]. Disponível: http://www.anvisa.gov.br/boletim_tecno/boletim_tecno_Junho_2011/PDF/Luvas\%20Cir\%C3\%BArgicas\%20 e\%20Luvas\%20de\%20Procedimentos_Considera\%C3\%A7\%C3\%B5es\%20sobre\%20o\%20uso.pdf

18. Conselho Regional de Enfermagem de São Paulo (COREN-SP). Parecer COREN-SP 042/2014-CT. Ementa: utilização de luvas de procedimentos para aplicação de vacina. São Paulo; 2014.

19 Santana JCB, de Sousa MA, Soares HC, Avelino KSA. Fatores que influenciam e minimizam os erros da administração de medicamentos pela equipe de enfermagem. Enferm. rev. [Internet] 2012; 15(1) [acesso em 28 fev 2016]. Disponível: http://periodicos.pucminas.br/index.php/enfermagemrevista/article/view/3300/3657

20. Rosa MB, Perini E, Anacleto TA, Neiva HM, Bogutchi T. Erros na prescrição hospitalar de medicamentos potencialmente perigosos. Revista de Saúde Pública. [Internet] 2009; 43(3) [acesso em 28 fev 2016]. Disponível: http://dx.doi.org/10.1590/S0034-89102009005000028

21. Veloso IR, Telles Filho PCP, Durão AMS. Identificação e análise de erros no preparo de medicamentos em uma unidade pediátrica hospitalar. Rev. Gaúcha Enferm. [Internet] 2011; 32(1) [acesso em 28 fev 2016]. Disponível: 
http://dx.doi.org/10.1590/S1983-14472011000100012

22. Cardoso SR, Pereira LS, e Souza ACS, Tipple AFV, Pereira MS, Junqueira ALN. Anti-sepsia para administração de medicamentos por via endovenosa e intramuscular. Rev. Eletr. Enf. [Internet] 2006; 8(1) [acesso em 28 fev 2016]. Disponível: https://dx.doi.org/10.5216/ree.v8i1.940

23. Lippert WC, Wall EJ. Optimal intramuscular needle-penetration depth. Pediatrics: Official Journal of the American Academy of Pediatrics. [Internet] 2008; 122(3): 556-63.

24. Hockenberry MJ, Wilson D. WONG, Fundamentos de Enfermagem Pediátrica. $9^{a}$ ed. Rio de Janeiro: Elsevier; 2014.

25. de Souza ECC, Bueno AAB, Fassarella CS. Segurança do paciente no ambiente hospitalar: os avanços na prevenção de eventos adversos no sistema de medicação. Revista Rede de Cuidados em Saúde. [Internet] 2013; 7(1) [acesso em 28 fev 2016]. Disponível: http://publicacoes.unigranrio.br/index.php/rcs/article/viewFile/1897/907

26. Miasso AI, Cassiani SHB. Erros na administração de medicamentos: divulgação dos conhecimentos e identificação do paciente como aspectos relevantes. Rev. esc. enferm. USP. [Internet] 2000; 34(1) [acesso em 28 fev 2016]. Disponível: http://dx.doi.org/10.1590/S0080-62342000000100003

27. Malagutti W, Roegrs H. Terapia Intravenosa: Atualidades. São Paulo: Martinari; 2012. 